Received: 24 January 2017

Accepted: 5 June 2017

Published online: 18 July 2017

\section{Cell-type specific differences in promoter activity of the ALS-linked C9orf72 mouse ortholog}

Abraham J. Langseth ${ }^{1}$, Juhyun Kim ${ }^{1}$, Janet E. Ugolino ${ }^{1,2}$, Yajas Shah ${ }^{1,2}$, Ho-Yon Hwang ${ }^{1,2}$, Jiou Wang $^{1,2}$, Dwight E. Bergles ${ }^{1}$ \& Solange P. Brown ${ }^{1}$

A hexanucleotide repeat expansion in the C9orf72 gene is the most common cause of inherited forms of the neurodegenerative disease amyotrophic lateral sclerosis (ALS). Both loss-of-function and gainof-function mechanisms have been proposed to underlie this disease, but the pathogenic pathways are not fully understood. To better understand the involvement of different cell types in the pathogenesis of ALS, we systematically analyzed the distribution of promoter activity of the mouse ortholog of C9orf72 in the central nervous system. We demonstrate that C9orf 72 promoter activity is widespread in both excitatory and inhibitory neurons as well as in oligodendrocytes and oligodendrocyte precursor cells. In contrast, few microglia and astrocytes exhibit detectable C9orf 72 promoter activity. Although at a gross level, the distribution of C9orf72 promoter activity largely follows overall cellular density, we found that it is selectively enriched in subsets of neurons and glial cells that degenerate in ALS. Specifically, we show that C9orf72 promoter activity is enriched in corticospinal and spinal motor neurons as well as in oligodendrocytes in brain regions that are affected in ALS. These results suggest that cell autonomous changes in both neurons and glia may contribute to C9orf72-mediated disease, as has been shown for mutations in superoxide dismutase-1 (SOD1).

A hexanucleotide repeat expansion mutation in chromosome 9 open reading frame 72 (C9orf72) is the most common known genetic cause of amyotrophic lateral sclerosis (ALS), a fatal neurodegenerative disease characterized by the progressive loss of corticospinal, brainstem and spinal motor neurons. A C 9 orf 72 mutation underlies approximately $40 \%$ of familial and $5 \%$ of sporadic ALS cases ${ }^{1-3}$. This hexanucleotide expansion is also found in approximately $10 \%$ of cases of a second neurodegenerative disease, frontotemporal dementia (FTD), a common cause of dementia in middle-aged patients ${ }^{3-5}$. Mutations in C9orf72 are a rare risk factor for several additional neurologic and psychiatric disorders including Alzheimer's disease, Parkinson's disease, Huntington's disease phenocopy patients, multiple system atrophy, depressive pseudodementia, bipolar disorder, and schizophrenia ${ }^{6-16}$. However, it is not known how this genetic mutation leads to these cell type-specific neurodegenerative disorders.

Both loss-of-function and gain-of-function mechanisms have been proposed to mediate C9orf72-linked $\mathrm{ALS}^{17,18}$. Several studies have demonstrated decreased C9orf 72 transcript and protein levels in patients with ALS and FTD ${ }^{19-23}$. Deletion of the mouse ortholog of the C9orf72 gene (3110043O21Rik, referred to here as C9orf72) has been reported to shorten lifespan and induce modest motor deficits in some, but not all mouse models, and cause profound dysregulation of the immune system ${ }^{24-29}$. Gain-of-function toxicity of the repeat expansion induced by sense and anti-sense RNA transcripts, as well as dipeptide proteins generated through repeat-associated non-ATG (RAN) translation, are also thought to contribute to neurodegeneration ${ }^{17}, 18$. Several transgenic mouse lines recently developed using patient-derived gene constructs demonstrate that C9orf72 repeat expansions induce age-dependent accumulation of RNA foci and dipeptide repeat proteins, along with neurodegeneration and behavioral abnormalities that at least partially recapitulate human disease $26,28,30,31$. Although the complex mechanisms underlying C9orf72-related disease have not been resolved, understanding the expression pattern of $C 9$ orf72 in the central nervous system (CNS) is not only important for understanding the pathogenesis of ALS, but is also relevant to the wide spectrum of $C 9$ orf72-associated diseases.

${ }^{1}$ Solomon H. Snyder Department of Neuroscience, Johns Hopkins University School of Medicine, Baltimore, Maryland, 21205, USA. 'Department of Biochemistry and Molecular Biology, Bloomberg School of Public Health, Johns Hopkins University, Baltimore, Maryland, 21205, USA. Abraham J. Langseth and Juhyun Kim contributed equally to this work. Correspondence and requests for materials should be addressed to J.W. (email: jiouw@jhmi. edu) or D.E.B. (email: dbergles@jhmi.edu) or S.P.B. (email: spbrown@jhmi.edu) 
Although the signature of ALS is the loss of corticospinal, brainstem, and spinal motor neurons, multiple cell types have been shown to contribute to the pathogenesis of the disease. Non-neuronal cells including oligodendrocytes, astrocytes, and microglia are also critical players in the pathogenesis of ALS ${ }^{17}$. Although some progress has been made in understanding the cell-type specific expression of $C 9$ orf $72^{26,28,32}$, a comparison of the distribution of C9orf72 expression across different neuronal and glial cell types in relevant regions of the brain and spinal cord is still lacking. Whether C9orf72 promoter activity is specifically enriched in affected corticospinal neurons, spinal motor neurons, or oligodendrocytes in regions implicated in ALS is not yet fully understood.

Here, we systematically mapped the promoter activity of the mouse ortholog of C9orf 72 in a genetically engineered strain of mice containing a targeted $L a c Z$ insertion under the control of the C9orf72 native promoter. Through quantitative comparisons among different types of neurons and glial cells labelled with retrograde neuronal tracers and cell type-specific markers, we demonstrate that mouse C9orf72 promoter activity, although widespread throughout the brain and spinal cord, is specifically enriched in corticospinal and spinal motor neurons and in oligodendrocytes, subsets of cells known to undergo degeneration in ALS, in regions affected by ALS. In contrast, C9orf72 promoter activity was detected in only a small percentage of microglial cells and even fewer astrocytes. Thus, these data suggest that, despite widespread expression, C9orf72 promoter activity reflects the patterns of degeneration typically seen in this disease, consistent with direct cell autonomous toxicity.

\section{Results}

The distribution of C9orf72 promoter activity and cellular density are highly correlated in the CNS. Mice have a single gene, $3110043 \mathrm{O} 21 \mathrm{Rik}$ (here referred to as C9orf72), which is orthologous to human

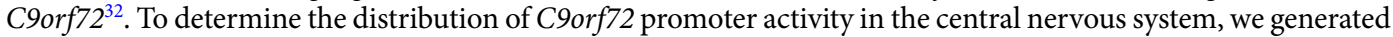
chimeric mice using several mouse embryonic stem cell lines heterozygous for an allele with a LacZ insertion in the C9orf72 locus generated by the Knock Out Mouse Project (KOMP) ${ }^{29,32-35}$. The LacZ insertion results in deletion of exons 2-6 of the mouse C9orf72 gene, producing a knockout allele (Supplementary Fig. 1a). Analysis of RNA-sequencing (RNA-seq) data from mice generated using the same targeting cassette and embryonic stem (ES) cell background indicates that the transcript structure is largely maintained between wild type (WT) and C9orf72 ${ }^{\text {LacZl+ }}$ mice $^{28}$ (Supplementary Fig. 1b). Although WT transcripts have two alternative starts, exon 1a or $1 \mathrm{~b}$, while the $L a c Z$ allele appears to use only exon $1 \mathrm{a}$, there is no reported evidence for differential usage of exon $1 \mathrm{a}$ and $\mathrm{lb}$ among different cell types indicating that the $L a c Z$ reporter likely reflects the pattern of the wild type gene expression. The heterozygous mice have a normal phenotype until six months of age, after which a fraction of $C 9$ orf $72^{\text {LacZ/+ }}$ mice exhibit an age-dependent decrease in survival, with approximately $20 \%$ of the heterozygotes dead by 600 days $^{29}$. We therefore used young, six to eight-week-old heterozygotes to assess the distribution of $L a c Z$ as a reporter for C9orf72 promoter activity.

We confirmed that C9orf72 promoter activity was not limited to areas known to degenerate in ALS and FTD. $\mathrm{X}$-gal staining to assess regions of $\beta$-galactosidase ( $\beta$-gal) expression (encoded by LacZ) revealed widespread promoter activity throughout the brain and spinal cord. The regions with the most intense X-gal signals in the brain corresponded to regions with high cell density, such as the dentate gyrus of the hippocampus and the granular layer of the cerebellum (Fig. 1a). Regions with the weakest signals corresponded to areas with low cell densities such as the molecular layer of the cerebellum and the corpus callosum (Fig. 1a). We also observed broad X-gal staining in primary motor cortex (Fig. 1a) and in the spinal cord (Fig. 1b). These results suggest that the distribution of $C 9$ orf 72 promoter activity largely follows cellular density across brain regions. To more directly test this hypothesis, we compared the distribution of cells stained with the nuclear marker, DAPI, and an antibody specific to $\beta$-galactosidase ( $\beta$-gal; Supplementary Fig. $1 \mathrm{c})$ and found that the distribution of $\beta$-gal signal correlated with the overall cellular density across layers in primary motor cortex, primary somatosensory cortex, and spinal cord grey matter (Fig. 1c-d). Together, these results indicate that C9orf72 promoter activity is widely distributed in the CNS, consistent with that reported in previous studies ${ }^{26,27,32}$, and largely correlates with overall cellular density.

The C9orf72 promoter is active in both excitatory and inhibitory neurons. Although at a macroscopic level, C9orf72 promoter activity was correlated with overall cellular density throughout the brain and spinal cord, enrichment or reduction of $C 9$ orf 72 promoter activity in specific regions of the neocortex or spinal cord or within specific cell types in each region would go undetected at this level of analysis. Therefore, we compared the distribution of C9orf72 promoter activity in neurons in primary motor cortex, a cortical region which undergoes degeneration in ALS, as well as primary somatosensory cortex (see Methods). C9orf72 promoter activity was observed in more than $80 \%$ of the neurons labelled with antibodies to NeuN in layer 5 (L5) of primary motor cortex, which contains corticospinal neurons known to degenerate in ALS (Fig. 2a,b). Unexpectedly, neurons in layer $2 / 3$ (L2/3) of primary somatosensory cortex, which consists primarily of excitatory corticocortical projection neurons, and L5 of primary somatosensory cortex, exhibited a similar distribution of 69 orf 72 promoter activity (Fig. 2a,b). Due to the high density of small $\beta$-gal-positive puncta in our samples, it is possible that we detected C9orf72 promoter activity in such a large number of neurons spuriously. To assess whether the distribution of the $\beta$-gal signal was specific to these neurons, we compared the percentage of $\beta$-gal-positive neurons detected before and after inverting the $\beta$-gal channel relative to the NeuN channel in each analyzed image. Following channel inversion, the percentage of $\beta$-gal-positive neurons significantly decreased, indicating that the relationship between the distribution of $\beta$-gal-positive puncta and the distribution of neurons is greater than would be expected by chance (Supplementary Fig. 2). These data demonstrate that the overall distribution of C9orf72 promoter activity in neurons is similar between primary motor cortex and primary sensory cortex when specific cell types are not taken into account.

Although C9orf72 transcripts have been identified in excitatory cortical projection neurons and spinal motor neurons ${ }^{26,32}$, whether C9orf72 promoter activity is found in inhibitory neurons is not known. To test for C9orf72 promoter activity in inhibitory neurons, we first analysed neurons in layer 1 (L1) of the cortex, which 
a
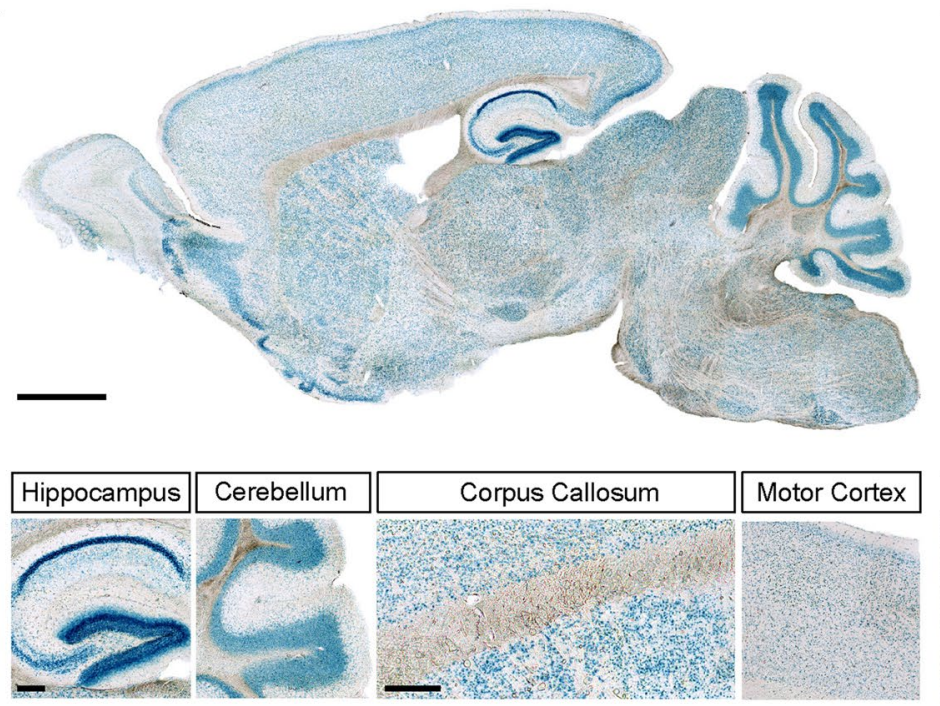

C
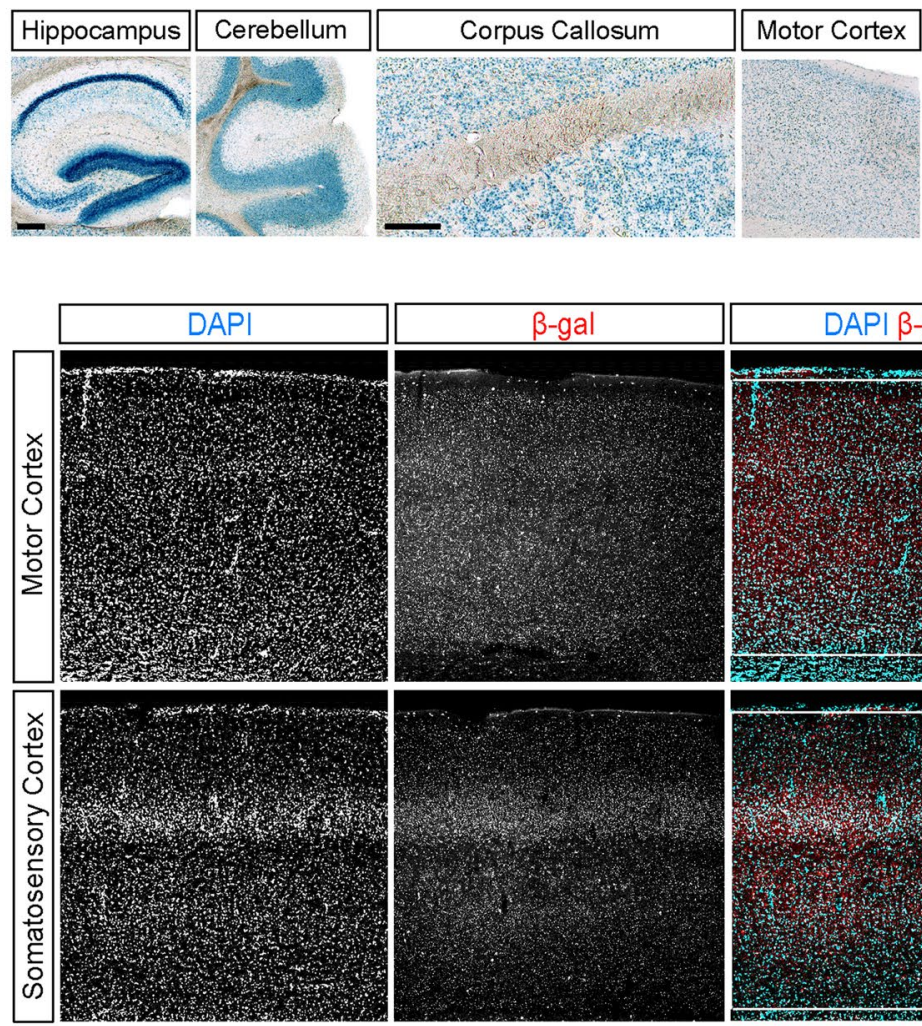
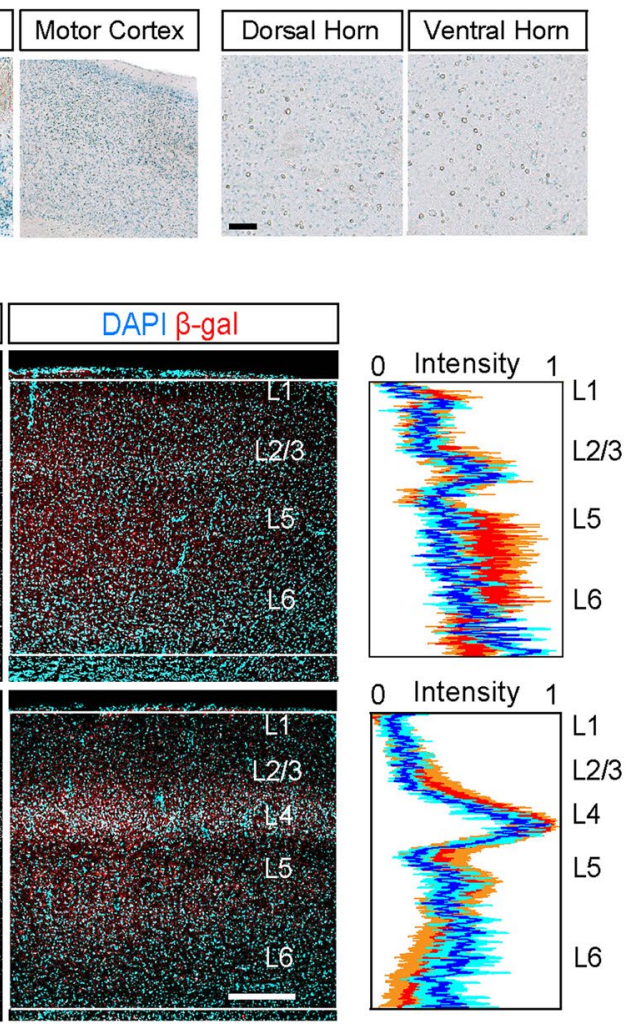

0 Intensity 1

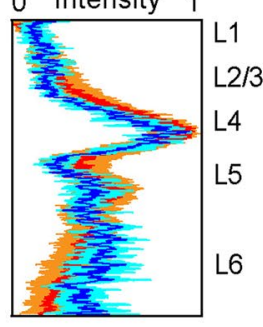

d
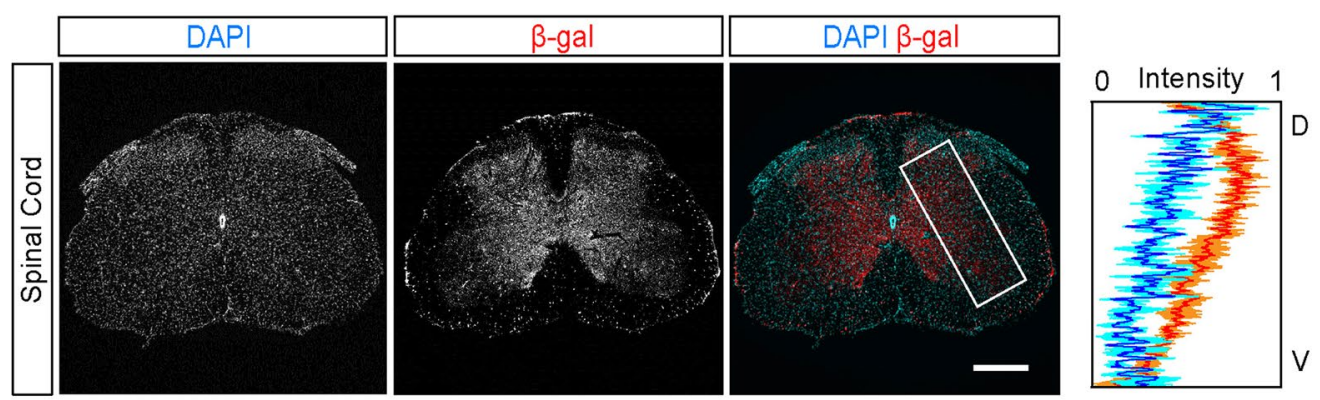

Figure 1. The distribution of C9orf72 promoter activity follows overall cellular density in the central nervous system. (a) The overall distribution of C9orf72 promoter activity in a parasagittal brain section from a C9orf72 $2^{\text {LacZ/+ }}$ mouse revealed using X-gal staining. C9orf72 promoter activity was high in brain regions with high cell density (dentate gyrus of the hippocampus, cerebellar granule cell layer, two left panels), low in regions with low cell density (cerebellar molecular cell layer and corpus callosum, middle panels), and medium in motor cortex (rightmost panel). (b) The overall distribution of C9orf 72 promoter activity in a coronal section of the lumbar spinal cord. $\mathrm{X}$-gal staining was seen in both the dorsal and ventral horns (left and right panels) (c) Representative images showing the distribution of immunofluorescence staining for $\beta$-gal in primary motor (top) and somatosensory (bottom) cortex of C9orf72 $2^{\mathrm{LaCZ} /+}$ mice concurrently labelled with the nuclear stain, DAPI. The intensities of the $\beta$-gal (red) and DAPI (blue) signals were summed along the horizontal axis in the region indicated by the white boxes $(\mathrm{n}=3$ mice) and the intensity values plotted (mean \pm SEM). (d) A similar analysis was performed for lumber spinal cord ( $n=3$ mice). Scale bars: (a) $1 \mathrm{~mm}$, insets $100 \mu \mathrm{m}$, (b) $200 \mu \mathrm{m}$, insets $100 \mu \mathrm{m}$ and (c,d) $300 \mu \mathrm{m}$. 
a

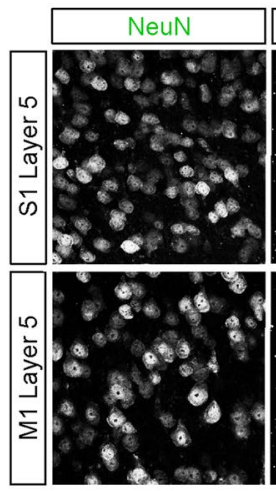

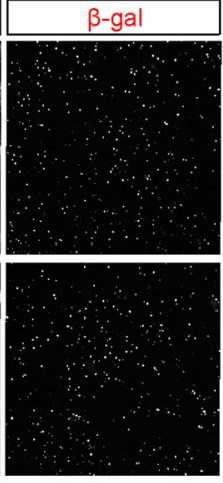

C

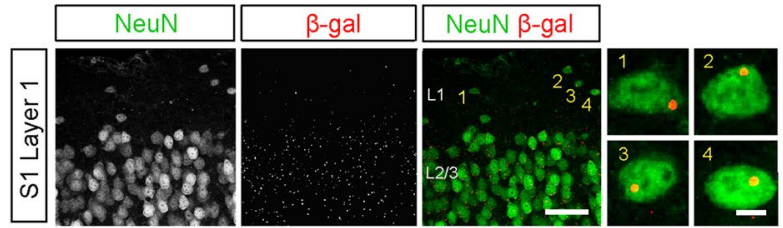

e
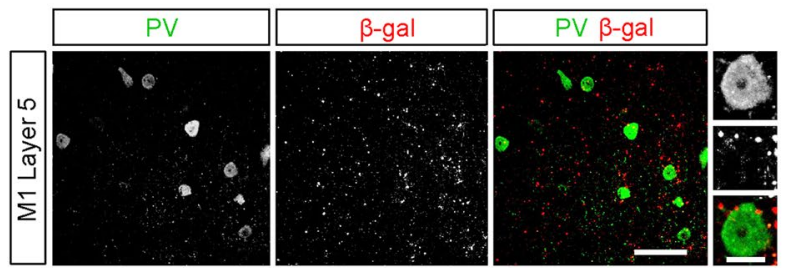

b

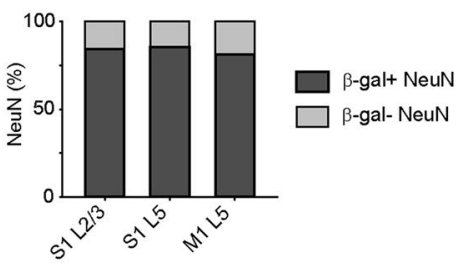

d

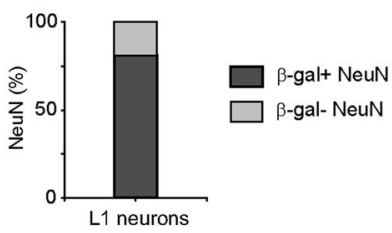

f

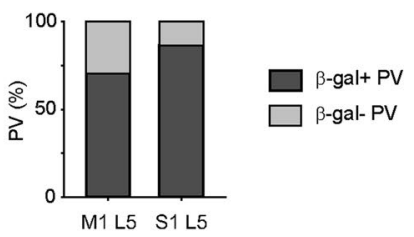

Figure 2. C9orf72 promoter activity is widespread in inhibitory neurons. (a) Representative images of layer 5 (L5) of primary somatosensory cortex $(\mathrm{S} 1$, top $)$ and primary motor cortex $(\mathrm{M} 1$, bottom $)$ from C9orf $72^{\text {LacZ/+ }}$ mice stained for NeuN (green) and $\beta$-galactosidase ( $\beta$-gal; red). High magnification images show $\beta$-gal-positive puncta within NeuN-positive neurons (rightmost panels). Scale bars: $50 \mu \mathrm{m}$ and $10 \mu \mathrm{m}$. (b) The percentage of neurons containing $\beta$-gal puncta in layer $2 / 3$ and L 5 of somatosensory cortex and L5 of motor cortex (S1 L2/3: 362 of 429 cells, 84.4\%; S1 L5, 499 of 583 cells, 85.6\%; M1 L5: 425 of 523 cells, 81.3\%; $n=3$ mice for each group, $p=0.1378$, Chi-Square test). (c) Representative images of layer 1 (L1) of somatosensory cortex stained for NeuN (green) and $\beta$-gal (red). High magnification images showing $\beta$-gal-positive puncta within L1 neurons (rightmost panels). Scale bars: $50 \mu \mathrm{m}$ and $5 \mu \mathrm{m}$. (d) The percentage of $\mathrm{L} 1$ neurons containing $\beta$-gal puncta ( 26 of 32 cells, $81.3 \%, n=3$ mice). (e) Representative images of L5 of motor cortex stained for parvalbumin (PV, green) and $\beta$-gal (red). High magnification images show $\beta$-gal puncta within a PV interneuron (rightmost panels). Scale bars: $50 \mu \mathrm{m}$ and $10 \mu \mathrm{m}$. (f) The percentage of PV interneurons containing $\beta$-gal puncta in L5 of motor cortex and somatosensory cortex (M1 L5: 36 of 51 cells, 70.6\%; S1 L5: 32 of 37 cells, 86.5\%; $n=3$ mice for each group, $p=0.0789$, Chi-Square test).

are overwhelmingly GABAergic ${ }^{36,37}$. We found that $\beta$-gal-positive puncta were detected in more than $80 \%$ of $\mathrm{L} 1$ inhibitory interneurons (Fig. 2c-d). Similarly, we found that C9orf72 promoter activity was detected in approximately 70 to $85 \%$ of cortical parvalbumin-expressing (PV) inhibitory interneurons, the most common type of interneurons in the cortex ${ }^{37}$ (Fig. 2e,f), demonstrating that C9orf72 promoter activity is not limited to glutamatergic cortical projection neurons. Taken together, these data demonstrate that C9orf 72 promoter activity is also widespread in inhibitory interneurons.

C9orf72 promoter activity is enriched in corticospinal and spinal motor neurons. The hallmark of ALS is progressive degeneration of corticospinal and spinal motor neurons. A previous study reported that the majority of layer 5 (L5) cortical neurons exhibiting C9orf72 promoter activity were CTIP2-positive ${ }^{32}$, suggesting that sub-cerebral projection neurons, which include the corticospinal neurons (CSNs) that degenerate in ALS, express C9orf72. To further test this hypothesis, we compared the distribution of C9orf72 promoter activity in retrogradely labelled CSNs and in unlabeled NeuN-positive neurons intermingled within L5 of motor cortex (Fig. 3a,b). A significantly higher percentage of CSNs exhibited $\beta$-gal-positive puncta than unlabeled neighboring neurons, indicating that C9orf72 promoter activity is specifically enriched in corticospinal neurons (Fig. 3c,d; $p<0.0001$, Chi-Square test). Similarly, a significantly higher percentage of choline acetyltransferase (ChAT)-immunoreactive spinal motor neurons exhibited detectable C9orf72 promoter activity than either ChAT-immunonegative neurons intermingled within the same region of the ventral horn or ChAT-immunonegative neurons in the dorsal horn of the spinal cord (Fig. 3e,f; $p<0.0001$, Chi-Square test). Together, these results indicate that $C 9$ orf 72 promoter activity is specifically enriched in both retrogradely 

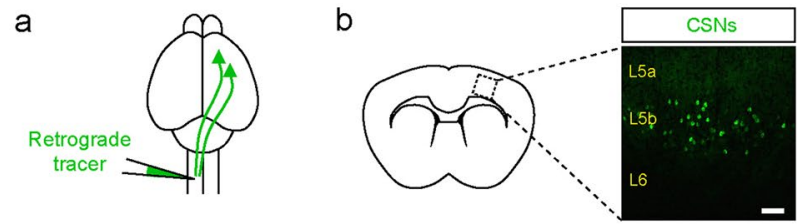

C
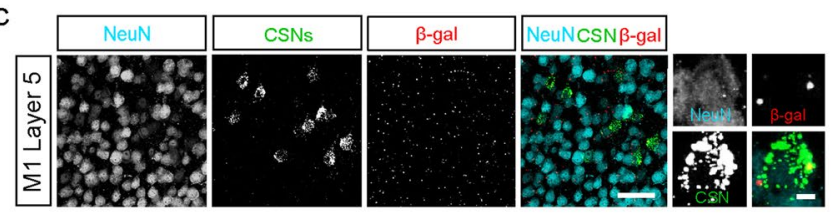

e
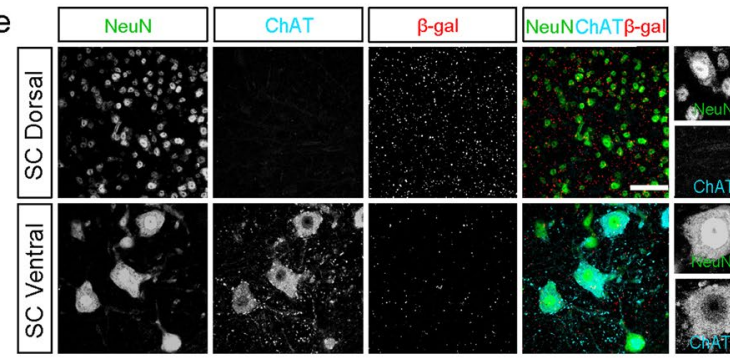

d

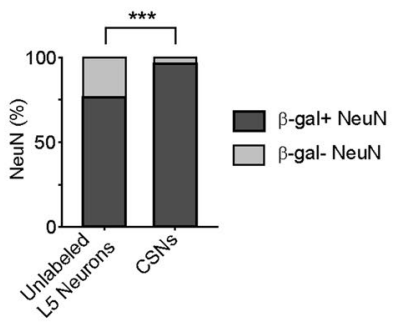

f

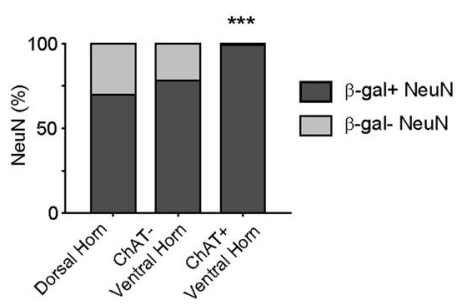

Figure 3. C9orf72 promoter activity is enriched in corticospinal and spinal motor neurons. (a) Schematic of the experimental design. Green fluorescent retrograde tracers were injected into the contralateral cervical spinal cord to retrogradely label corticospinal neurons in $\mathrm{C}^{\circ} \mathrm{orf7} 72^{\mathrm{LacZ} /+}$ mice. (b) Retrogradely labelled corticospinal neurons are shown in layer 5 (L5) of primary motor cortex. Scale bar: $100 \mu \mathrm{m}$. (c) Representative images of L5 of motor cortex showing NeuN-positive neurons (cyan), retrogradely labelled corticospinal neurons (green) and $\beta$-gal staining (red). High magnification images show $\beta$-gal puncta within a corticospinal neuron (rightmost panels). Scale bars: $50 \mu \mathrm{m}$ and $5 \mu \mathrm{m}$. (d) The percentage of unlabeled NeuN-positive neurons and of corticospinal neurons (CSNs) containing $\beta$-gal puncta in L5 of motor cortex (Unlabeled NeuN-positive neurons: 1357 of 1771 cells, $76.6 \%$; CSNs: 132 of 137 cells, $96.4 \% ; n=5$ mice for each group, $p<0.0001$, ChiSquare test). Unlabeled NeuN-positive neurons and retrogradely labelled corticospinal neurons were analyzed from the same images. (e) Representative images of the dorsal (top) and ventral horn (bottom) of the spinal cord immunostained for NeuN (green), Choline acetyltransferase (ChAT; cyan), and $\beta$-gal (red). High magnification images show $\beta$-gal puncta within NeuN-positive neurons in the dorsal horn (top right) and a ChAT-positive neuron in the ventral horn (bottom right). Scale bars: $50 \mu \mathrm{m}$ and $10 \mu \mathrm{m}$. (f) The percentage of ChAT-negative and ChAT-positive neurons containing $\beta$-gal puncta in the dorsal and ventral horn of spinal cord (dorsal neurons: 734 of 1050 cells, 69.9\%; ventral ChAT-negative neurons: 61 of 78 cells, 78.2\%; ventral ChAT-positive neurons: 113 of 114 cells, $99.1 \% ; n=5$ mice for each group; $p<0.0001$, Chi-Square test).

labelled corticospinal neurons and cholinergic spinal motor neurons, neuronal types that are specifically vulnerable in ALS.

C9orf72 promoter activity in Purkinje cells and cerebellar granule cells. C9orf72-mediated disease is notable for RNA foci and neuronal inclusions found in the cerebellum ${ }^{38-41}$. RAN-translated polypetides stemming from the hexanucleotide repeat expansion have been detected in Purkinje cells and granule cells ${ }^{40}$. Furthermore, prior studies have detected high levels of C9orf 72 expression in the cerebellum ${ }^{23,42}$. Here we assessed C9orf72 promoter activity in both Purkinje cells and granule cells in the cerebellum. Consistent with the X-gal staining showing intense signal in the cerebellum (Fig. 1a), we detected C9orf72 promoter activity in a large percentage of both Purkinje cells and cerebellar granule cells (Fig. 4). Overall, 41.2\% of Purkinje cells and $55.8 \%$ of granule cells had detectable C9orf72 promotor activity ( $p=0.0116$, Chi-Square test), indicating that C9orf72 promoter activity is relatively enriched in the granule cell layer. These data provide additional evidence for cell-type specific enrichment of C9orf72 promoter activity in distinct cell types in affected brain regions in C9orf72-mediated disease.

Cell-type and region specific regulation of C9orf72 promoter activity in glial cells. Glial cells play important roles in the pathophysiology of ALS. Astrocytes undergo reactive changes in ALS, although their role in the initiation and progression of C9orf72-mediated ALS remains to be elucidated ${ }^{17,43-45}$. While a prior study reported that spinal cord astrocytes did not express $C 9$ orf $72^{32}$, RNA sequencing experiments detected low levels of $C 9$ orf 72 transcripts in mouse $\mathrm{e}^{28,46}$ and human astrocytes ${ }^{47}$. Consistent with the RNA sequencing data, we found that approximately $20 \%$ of $\mathrm{S} 100 \beta$-positive astrocytes in the spinal cord had detectable C9orf 72 promoter activity (Fig. 5a-c). Interestingly, almost no S100ß-positive astrocytes in the cortex exhibited detectable C9orf72 promoter activity (Fig. 5a-c). These data indicate that far fewer astrocytes than neurons express C9orf72, and that $C 9$ orf 72 promoter activity is not only regulated in a cell-type specific manner but also in a region dependent way. 


\section{a}
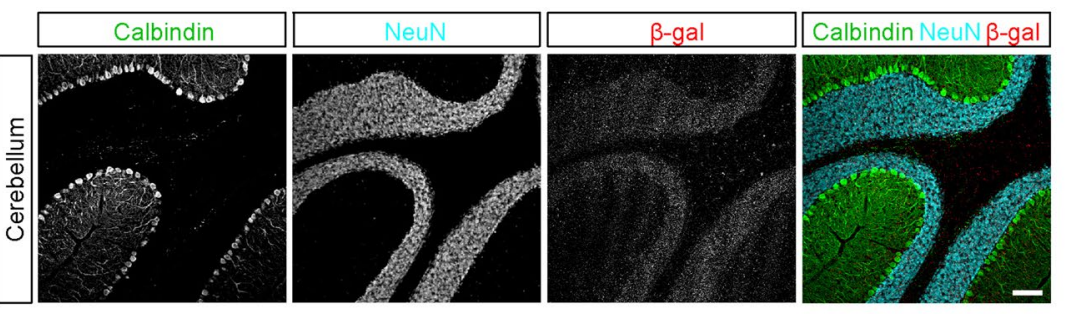

b
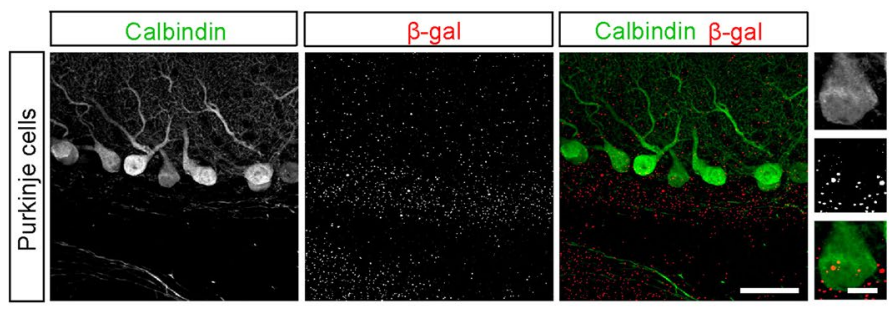

C

d

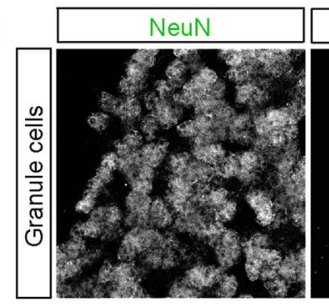

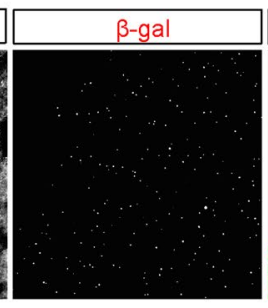
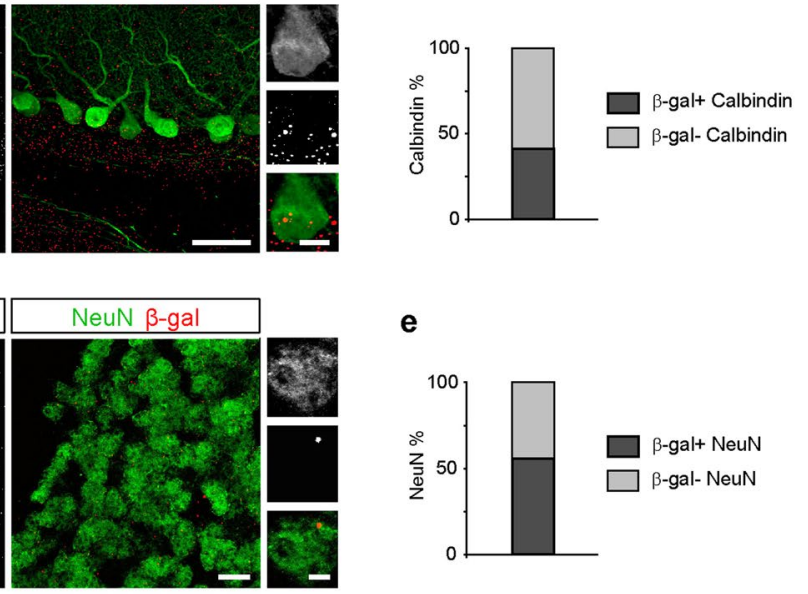

e

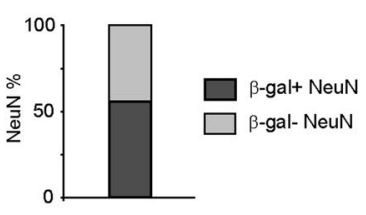

Figure 4. C9orf72 promoter activity in Purkinje cells and granule cells of the cerebellum. (a) Representative images of the cerebellum showing calbindin-positive Purkinje cells (green), NeuN-positive granule cells (cyan), and $\beta$-gal staining (red). Scale bar: $100 \mu \mathrm{m}$. (b) Representative images of calbindin-positive Purkinje cells (green) and $\beta$-gal (red). Scale bars: $50 \mu \mathrm{m}$ and $10 \mu \mathrm{m}$. (c) The percentage of Purkinje cells containing $\beta$-gal puncta ( 35 of 85 cells, $41.2 \%, n=3$ mice). (d) Representative images of NeuN-positive granule cells (green) and $\beta$-gal (red). Scale bars: $10 \mu \mathrm{m}$ and $2 \mu \mathrm{m}$. (e) The percentage of granule cells containing $\beta$-gal puncta: (306 of 548 cells, $55.8 \% ; n=3$ mice).

Recent studies have demonstrated that $C 9$ orf 72 plays an important role in regulating the immune system ${ }^{24,25,28}$ and have reported high levels of C9orf72 transcript expression in bulk cellular populations enriched for microglia ${ }^{28,46}$. In contrast, a prior study detected C9orf72 promoter activity in only a small subset of microglia in the anterior horn of the spinal cord ${ }^{32}$. Similarly, we found that only a small proportion of Iba1-immunoreactive microglia in the cortex and spinal cord had detectable C9orf72 promoter activity (Fig. 6a-c). These findings suggest that the level of expression of $C 9$ orf 72 varies substantially across the population of microglial cells in the brain and spinal cord, with only a few cells expressing high levels and the majority expressing low or undetectable levels.

Oligodendrocytes provide metabolic support critical for neuronal health particularly for long axons. The degeneration of oligodendrocytes seen in ALS patient tissue and in mouse models of the disease suggests that these cells play an important role in the selective vulnerability of specific neuronal populations in ALS ${ }^{48-51}$. Removal of the ALS mutation $S O D 1^{G 37 R}$ from the oligodendrocyte lineage significantly delays disease onset and extends the lifespan of ALS model mice ${ }^{52}$, providing further support for an important contribution of oligodendrocyte dysfunction in the disease. Although C9orf72 expression has been reported in oligodendrocytes ${ }^{26,28}$, the distribution of C9orf72 promoter activity in the cortex and spinal cord is still poorly understood. We found that more than $25 \%$ of CC1-immunoreactive oligodendrocytes in layer 5 of primary somatosensory cortex and of primary motor cortex exhibited C9orf72 promoter activity. Interestingly, we detected $\beta$-gal puncta in a significantly greater percentage of CC1-positive oligodendrocytes in motor cortex than primary somatosensory cortex (Fig. 7a,b; $p=0.014$, Chi-Square test), indicating enrichment of C9orf72 expression in cortical regions which undergo the most profound degeneration in ALS. An even greater percentage of oligodendrocytes in the spinal cord exhibited detectable C9orf72 promoter activity (Fig. 7a,c); the highest percentage of $\beta$-gal-positive, CC1-positive oligodendrocytes was found in the ventral white matter of the spinal cord, where almost $65 \%$ of oligodendrocytes were positive for $\beta$-gal (Fig. 7c; $p<0.0001$, Chi-Square test). C9orf72 promoter activity was also observed in approximately $20-35 \%$ of cortical NG2-immunoreactive oligodendrocyte precursor cells (OPCs; Fig. $7 \mathrm{~d}, \mathrm{e}$ ). More than $50 \%$ of OPCs of the spinal cord also exhibited C9orf72 promoter activity (Fig. $7 \mathrm{~d}, \mathrm{f}$ ). These data indicate that $C 90$ orf 72 promoter activity is detected in a much larger percentage of oligodendrocytes and OPCs than in astrocytes and microglia, and that C9orf72 promoter activity is specifically enriched in oligodendrocytes in regions thought to degenerate in ALS. 
a
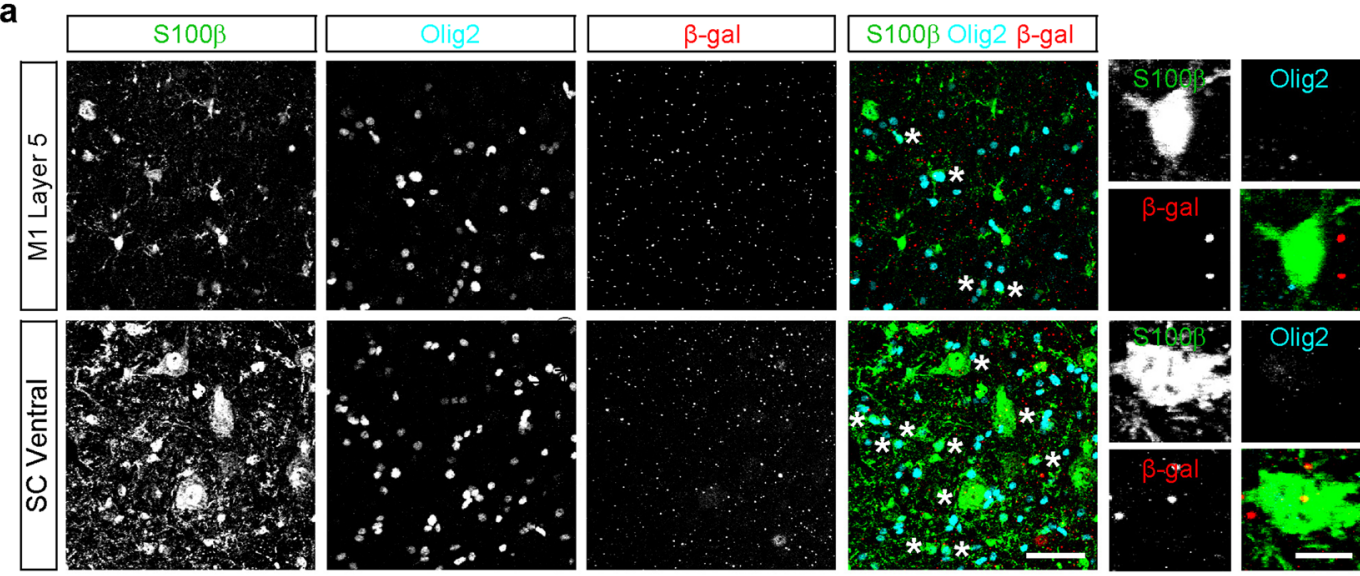

b

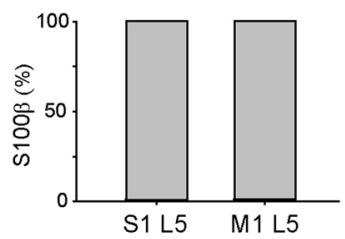

c

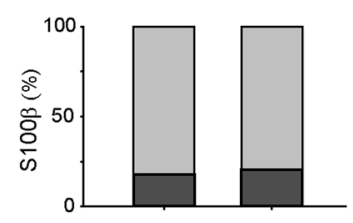

$\beta-g a l+S 100 \beta+$

$\beta$-gal- $S 100 \beta+$

Figure 5. Differential C9orf72 promoter activity in cortical and spinal cord astrocytes. (a) Representative images of layer 5 (L5) in primary motor cortex (M1, top) and the ventral horn of the spinal cord (SC, bottom) of $C 90 r f 72^{\text {LacZ/+ }}$ mice immunostained for S1003-positive astrocytes (green), Olig2-positive oligodendrocyte lineage cells (cyan), and $\beta$-gal (red). Asterisks indicate S100 $\beta$-positive Olig2-positive oligodendrocyte lineage cells and $\mathrm{S} 100 \beta$-positive motor neurons which were excluded from the analysis. High magnification images show an astrocyte in motor cortex which lacks $\beta$-gal puncta (top right) and astrocytes in the spinal cord which contain $\beta$-gal puncta (bottom right). Scale bars: $50 \mu \mathrm{m}$ and $10 \mu \mathrm{m}$. Percentage of astrocytes containing $\beta$-gal puncta in L5 of primary motor and primary somatosensory cortex (b) L5 motor cortex: 1 of 92 cells, 1.1\%, L5 somatosensory cortex, 1 of 98 cells, $1.0 \%, n=3$ mice for each group, $p=0.9641$, Chi-Square test) and spinal cord (c) dorsal spinal cord: 15 of 84 cells, $17.9 \%$, ventral spinal cord: 16 of 78 cells, $20.5 \%, n=3$ mice for each group, $p=0.6676$, Chi-Square test).

\section{Discussion}

Genes that are mutated in ALS are widely expressed in the CNS, creating unique vulnerabilities in distinct cell types. To understand how these mutations alter cellular physiology we must define both the tissue and cellular expression patterns of these mutant genes. Here, we show that although the distribution of C9orf 72 promoter activity follows overall cellular density, there is striking enrichment in neuronal and glial cell types that degenerate in ALS. C9orf72 promoter activity was detected in a significantly higher percentage of both corticospinal and spinal motor neurons than in neighboring neuronal cell types. Similarly, we demonstrate that C9orf72 promoter activity was enriched in oligodendrocytes in cortical regions associated with ALS pathology compared to unaffected areas. In contrast to neurons and oligodendrocytes, we detected C9orf72 promoter activity in few astrocytes and microglia ${ }^{32}$. Together, these data indicate that the pattern of degeneration seen in ALS reflects the distribution of $C 9$ orf 72 promoter activity, suggesting that cell autonomous effects in these populations of neurons and glia may account for their loss.

The widespread expression of C9orf72 promoter activity we observed in this Neo-deleted KOMP mouse is consistent with the overall expression patterns reported in recent studies of the KOMP mouse ${ }^{26,32}$ as well as C9orf72 RNA expression data ${ }^{26-28,53}$, and indicates that the distribution of C9orf72 promoter activity grossly follows cellular density throughout the CNS. However, an analysis of specific cell types demonstrated that widely varying fractions of cells expressed C9orf72. For example, a large majority of both inhibitory and excitatory neurons exhibited C9orf72 promoter activity while a small minority of astrocytes did. The widespread expression of C9orf72 by interneurons which we demonstrate here is particularly interesting, given their pivotal role in regulating neuronal excitability and brain states, and dysfunction of cortical inhibitory neurons may contribute to the cortical hyperexcitability seen in patients with C9orf72 disease ${ }^{54,55}$

Our data also indicate that C9orf72 promoter activity is enriched in cell types and brain regions that undergo degeneration in ALS. A higher percentage of corticospinal neurons express C9orf72 than either neighboring neurons in motor cortex or neurons in L5 of somatosensory cortex. Similarly, a higher percentage of oligodendrocytes in L5 of motor cortex express C9orf72 than in L5 of somatosensory cortex. We also found that approximately $25-50 \%$ of oligodendrocytes and OPCs in the cortex and spinal cord expressed C9orf72. Importantly, we show that a greater fraction of oligodendrocytes in L 5 of motor cortex expresses C9orf72, providing further evidence that cell types that degenerate in ALS express C9orf72 more widely than other cell types in the CNS. Together 
a
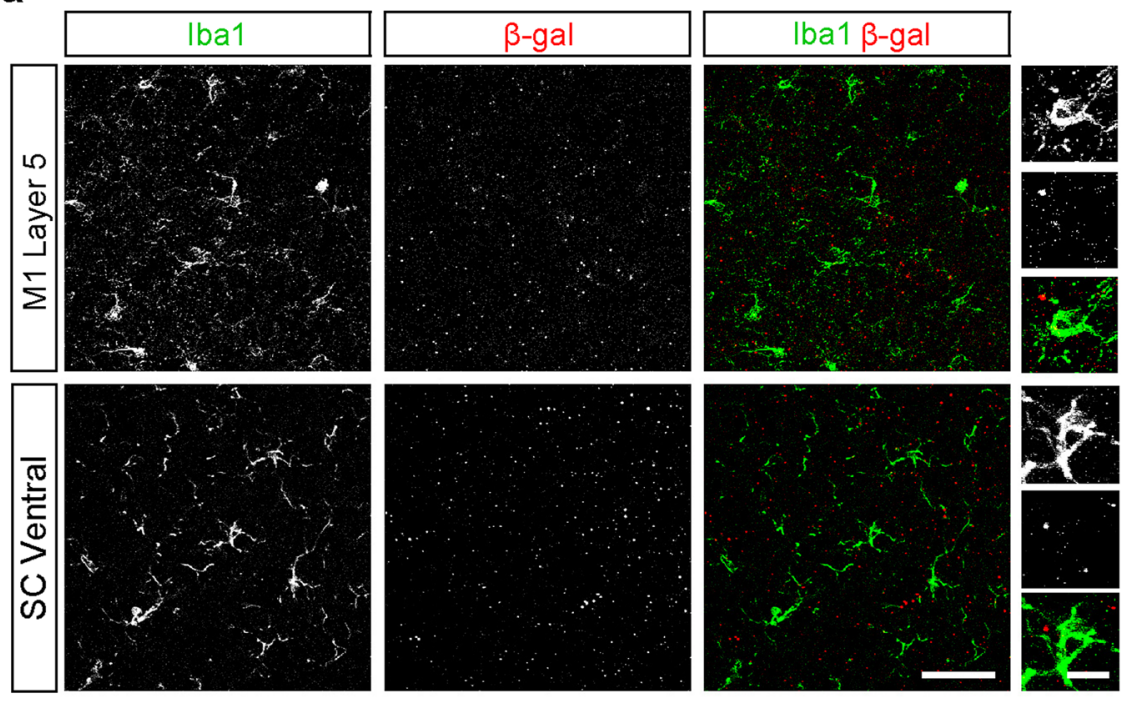

b

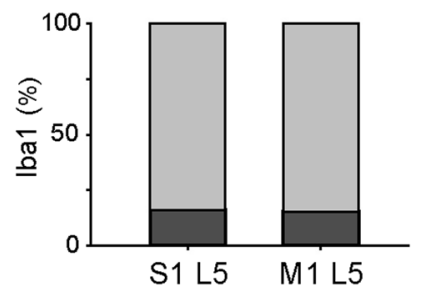

C

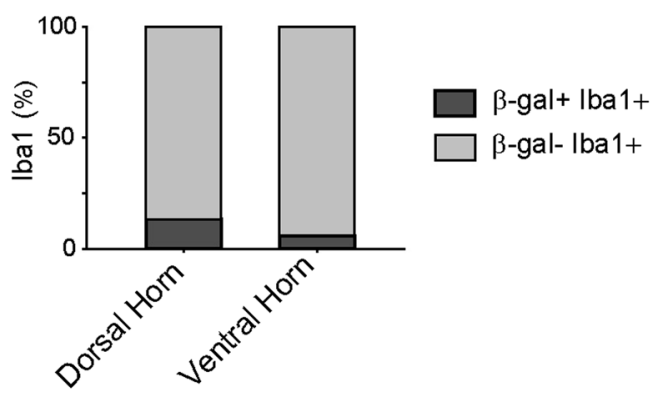

Figure 6. C9orf 72 promoter activity is detected in few microglia. (a) Representative images of layer 5 (L5) of primary motor cortex (M1, top) and the ventral horn of the spinal cord (SC, bottom) of C9orf72 ${ }^{\mathrm{LacZ} /+}$ mice immunostained for Iba1-positive microglia (green) and $\beta$-gal (red). High magnification images show microglia which do not contain $\beta$-gal puncta in the motor cortex (top right) and the spinal cord (bottom right). Scale bars: $50 \mu \mathrm{m}$ and $10 \mu \mathrm{m}$. The percentage of microglia containing $\beta$-gal puncta in L5 of the primary motor and primary somatosensory cortex (b) L5 of somatosensory cortex: 16 of 127 cell, 16.0\%; L5 of motor cortex: 22 of 160 cells, $15.2 \% ; n=7$ mice for each group, $p=0.7749$, Chi-Square test) and the spinal cord (c; dorsal spinal cord: 14 of 106 cells, $13.2 \%$; ventral spinal cord: 2 of 34 cells, $5.9 \%, n=3$ mice for each group, $p=0.2427$, Chi-Square test).

with the evidence of widespread degeneration of oligodendrocytes in ALS patient tissue and mouse models ${ }^{48-51}$, and the finding that removal of the SOD ${ }^{\mathrm{G} 37 R}$ ALS mutant transgene selectively from oligodendrocyte lineage cells significantly delays disease onset and extends the lifespan of ALS model mice ${ }^{52}$, these data support the hypothesis that oligodendroglia are critical primary players in the selective degeneration of CNS regions in ALS. The pathological mechanisms associated with expression of mutated C9orf 72 may confer selective vulnerability on these cell types during the course of ALS and may help to explain the regional pattern of degeneration observed in ALS.

A hallmark of C9orf72-mediated disease is cerebellar pathology including RNA foci and neuronal inclusions $^{38-41}$. Prior studies have also detected high levels of C9orf72 expression in the cerebellum ${ }^{23,42}$. Consistent with this work, we detected C9orf72 promoter activity in both Purkinje cells and cerebellar granule cells. Interestingly, a higher percentage of granule cells exhibited detectable C9orf 72 promoter activity than neighboring Purkinje cells, contributing to the intense staining we detected in the granule cell layer. Although there are marked neuropathological findings in the cerebellum in C9orf72-mediated disease and high levels of C9orf72 expression, the relationship between these findings and any neurodegeneration remains unclear ${ }^{38,56}$.

In contrast to the enrichment of C9orf72 promoter activity that we detected in neurons and oligodendrocyte lineage cells, we found that very few astrocytes and microglia exhibited detectable C9orf 72 promoter activity. Furthermore, there were striking regional differences in expression. For example, almost no cortical astrocytes exhibited C9orf72 promoter activity while approximately $20 \%$ of the astrocytes in the spinal cord expressed C9orf72. These results are consistent with analyses of C9orf72 transcript expression, although an early study did not detect $L a c Z$ expression in spinal cord astrocytes in a similar mouse line ${ }^{24,26,28,32,46}$. Recent studies have demonstrated high levels of $C 9$ orf72 expression in enriched microglia cell populations $\mathrm{s}^{26,28,46}$. This result is surprising in view of the small percentage of spinal cord microglia previously found to exhibit C9orf72 promoter activity in the KOMP mouse ${ }^{32}$. Not only did we also detect C9orf72 promoter activity in only a small fraction of 
a
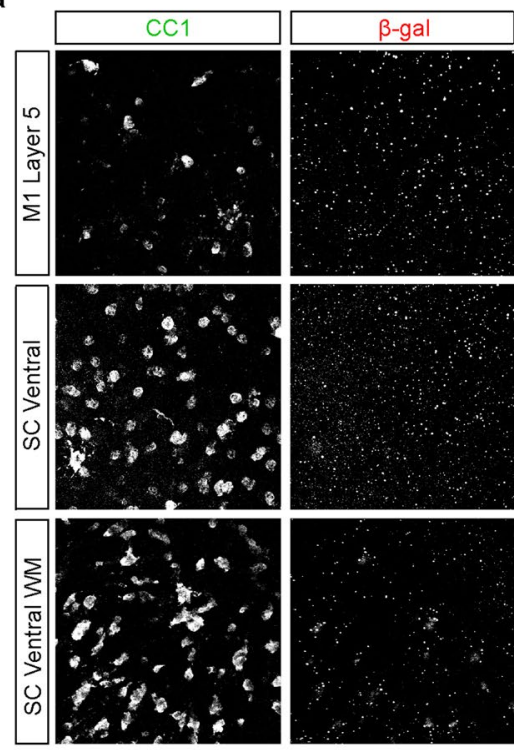

d

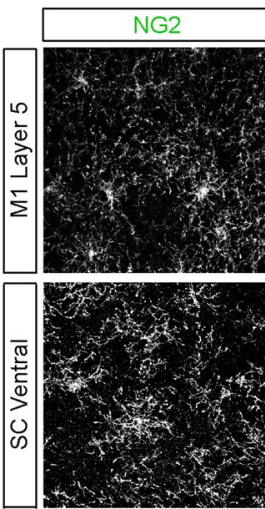

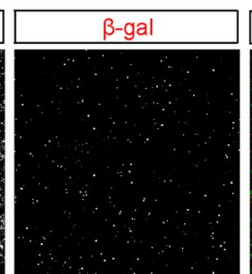

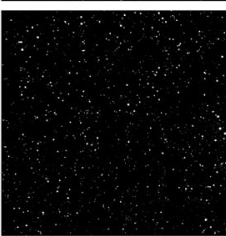

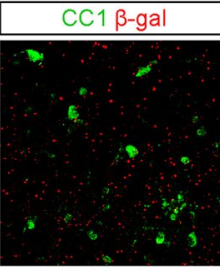
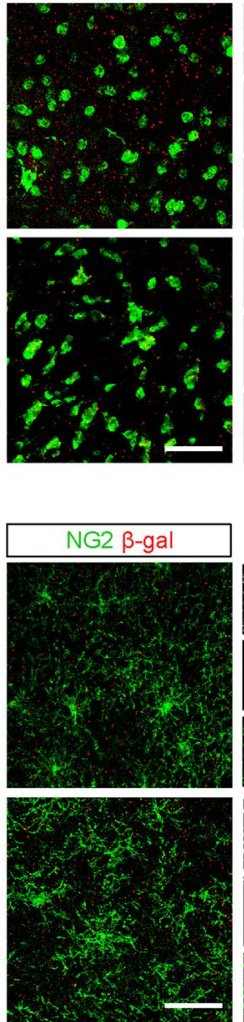
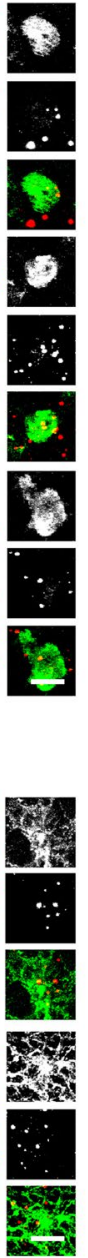
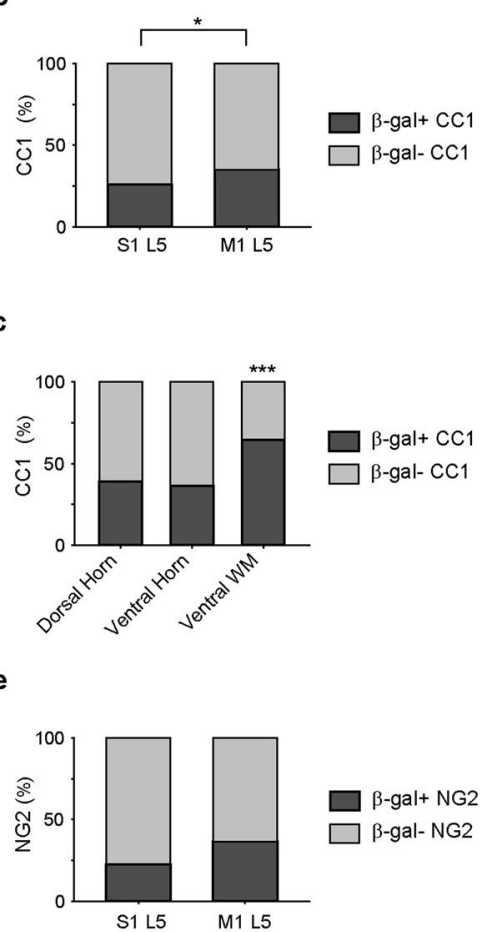

f

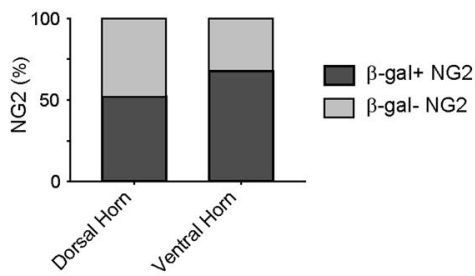

Figure 7. C9orf72 promoter activity in oligodendrocytes and OPCs is enriched in cortical regions associated with neurodegeneration in amyotrophic lateral sclerosis. (a) Representative images of layer 5 (L5) of the primary motor cortex (M1, top), ventral horn of the spinal cord (SC, middle), and the ventral white matter of the spinal cord (SC WM, bottom) of C9orf72 $2^{\mathrm{LacZ} /+}$ mice immunostained for CC1-positive oligodendrocytes (green) and $\beta$-gal (red). High magnification images show colocalization of $\beta$-gal puncta within oligodendrocytes (right panels).The percentage of oligodendrocytes containing $\beta$-gal puncta in L5 of primary somatosensory (S1) and motor (M1) cortex (b) S1 L5: 38 of 167 cells, 26.0\%; M1 L5: 82 of 234 cells, 35.0\%; $n=5$ mice for each group, $\mathrm{p}=0.0140$, Chi-Square test) and spinal cord (c) dorsal spinal cord: 209 of 533 cells, $39.2 \%$; ventral spinal cord: 191 of 525 cells, $36.4 \%$; spinal cord white matter: 283 of 438 cells, $64.6 \%, n=3$ mice for each group, $\mathrm{p}<0.0001$ Chi-Square) (d) Representative images of L5 of primary motor cortex (M1, top) and the ventral horn of the spinal cord (bottom) immunostained for NG2-positive oligodendrocyte precursor cells (OPCs; green) and $\beta$-gal (red). High magnification images show colocalization of $\beta$-gal puncta within oligodendrocytes (right panels). The percentage of OPCs containing $\beta$-gal puncta in L 5 of primary motor (M1) and primary somatosensory (S1) cortex (e) S1 L5: 21 of 92 cells, 22.6\%; M1 L5: 33 of 92 cells, 36.5\%; $n=5$ mice for each group, $p=0.0520$, ChiSquare test) and spinal cord (f) dorsal spinal cord: 39 of 75 cells, 52.0\%; ventral spinal cord: 44 of 65 cells, $67.7 \%$; $\mathrm{n}=3$ mice for each group, $\mathrm{p}=0.0594$, Chi-Square test). Scale bars: $50 \mu \mathrm{m}$ and $10 \mu \mathrm{m}$.

microglia in the spinal cord, we found that few microglia in the cortex exhibited detectable C9orf 72 promoter activity. It is possible that $C 9$ orf 72 transcript levels may depend on the activation state of a microglial cell, and that C9orf72 expression is rapidly upregulated when cells are dissociated from the brain for gene expression analysis. Recent work demonstrating that immune challenge of monocytes increases C9orf72 expression hints at this possibility, although unchallenged monocytes express higher levels of C9orf72 than microglia from fetal human brain tissue $^{23}$. Alternatively, there may be widely varying levels of expression in different microglial cells, with a small fraction of microglial cells expressing high levels of C9orf72 leading to high levels of transcript detection in bulk populations. Understanding the patterns of C9orf72 expression in combination with the distribution of the protein in different cell types represents an essential step in elucidating the mechanisms of dysfunction in CNS cells in the spectrum of $C 9$ orf 72 -associated diseases. Several studies have demonstrated decreased C9orf 72 transcript and protein levels in patients with ALS and $\mathrm{FTD}^{19-23}$, suggesting that haploinsufficiency may contribute to ALS 
pathogenesis. Our analysis of the patterns of C9orf72 expression suggests that the cell types that undergo degeneration in ALS, including corticospinal neurons, spinal motor neurons and oligodendrocytes, would be most affected by any $C 9$ orf 72 haploinsufficiency. However, the mechanisms underlying the range of phenotypes following C9orf72 deletions in mouse models remains to be elucidated ${ }^{24-29}$, and understanding this variability remains an essential step for uncovering the cellular mechanisms underlying ALS and other C9orf72-linked diseases.

\section{Methods}

Generation of C9orf $72^{\text {LacZ/+ }}$ mice. All experimental procedures were approved by the Johns Hopkins Animal Care and Use Committee and conducted in accordance with the guidelines of the National Institutes of Health and the Society for Neuroscience. The mice carrying a targeted deletion of C9orf72 and insertion of LacZ were generated as described ${ }^{29}$. In brief, several embryonic stem cell lines harboring an insertion that replaced exons 2-6 of the mouse C9orf72 gene (3110043O21Rik) with LacZ (National Institutes of Health Knockout Mouse Project) were used for blastocyst injection to generate chimeric mice which were then selected for germline transmission. The original embryonic stem cells had the genetic background of C57BL/6N-Atm1Brd and the derived mice were maintained on the C57BL/6 background. Male mice bearing the targeted allele were crossed with Sox2Cre recombinase transgenic female mice (Jackson Laboratory, 008454), maintaining the C57BL/6 background, to remove the LoxP-flanked neomycin selection cassette in all progeny. Subsequent breeding eliminated the Sox2Cre transgene from our mouse line. Six to eight-week-old Neo-deleted C 9 orf $72^{\mathrm{LacZ} /++}$ heterozygous mice were used for all experiments. The genotyping primers were the following: gaatggagatcggagcacttatgg (wild-type, forward), gccttagtaactaagcttgctgccc (wild-type, reverse), gcacaagctatgttcatttgg (KO, forward), gactaacagaagaacccgttgtg (KO, reverse).

Immunohistochemistry and X-galactosidase staining. Mice were deeply anesthetized with sodium pentobarbital $(100 \mathrm{mg} / \mathrm{kg})$ and perfused transcardially with $4 \%$ paraformaldehyde in $0.1 \mathrm{M}$ sodium phosphate buffer. Brain and spinal cord tissue was isolated and postfixed in this solution overnight at $4{ }^{\circ} \mathrm{C}$, then washed in phosphate buffer. Spinal cord tissues were cryoprotected in 30\% sucrose, and sectioned at $35 \mu \mathrm{m}$ thickness on a cryostat. Brain sections, $35 \mu \mathrm{m}$ thick, were prepared using a vibratome (VT-1000S, Leica). To obtain sections of motor cortex, the brain was mounted in coronal orientation on a $15^{\circ}$ ramp prior to cutting slices. To obtain sections of somatosensory cortex, the brain was mounted in parasagittal orientation on a $30^{\circ}$ ramp prior to cutting slices. Cerebellar slices were obtained in the parasagittal orientation, after mounting the cerebellum on a flat block. Free-floating sections were permeabilized with $0.3 \%$ Triton X-100 in $0.1 \mathrm{M}$ sodium phosphate buffer for at least $5 \mathrm{~min}$ and then blocked with $0.3 \%$ Triton X-100 and 5\% normal donkey serum in $0.1 \mathrm{M}$ sodium phosphate buffer (blocking solution) for at least $1 \mathrm{~h}$ at room temperature. When performing immunohistochemistry for calbindin, $5 \%$ normal goat serum was also added. Sections were then incubated with primary antibodies prepared in blocking solution overnight at $4{ }^{\circ} \mathrm{C}$ and then incubated with secondary antibodies in blocking solution for at least $2 \mathrm{~h}$ at room temperature. Primary antibodies used included the following: rabbit anti- $\beta$-galactosidase (1:5000; gift from Dr. Joshua Sanes, Harvard University, Cambridge, MA), guinea pig anti-NG2 (1:30,000; Dr. Dwight Bergles, Johns Hopkins University, Baltimore, MD), mouse anti-APC (CC1; 1:50; Millipore, Cat. No. OP80), guinea pig anti-Olig2 (1:20,000; gift from Dr. Ben Novitch, University of California, Los Angeles, CA), mouse anti-S100 $\beta$ (1:400; Sigma, Cat. No. S2532), goat anti-Iba1 (1:250; Novus, Cat. No. NB100-1028), mouse anti-NeuN (1:500; Millipore, Cat. No. MAB377), mouse anti-Parvalbumin (1:300; Swant, Cat. No. PV235), goat anti-ChAT (1:500; Millipore, Cat. No. AB144P) and chicken anti-calbindin D28 (1:100, EnCor Biotechnology Inc., Cat. No. CPCA-Calb). Secondary antibodies used, all raised in donkey except for the goat anti-chicken secondaries, included the following: Alexa Fluor 488-, 546-, and 647- as well as Cy2-, Cy3-, and Cy5-conjugated secondary antibodies to rabbit, guinea pig, mouse, and goat (1:2000; Invitrogen and Jackson ImmunoResearch). For $\mathrm{X}$-galactosidase staining, sections were incubated in $1 \mathrm{mg} / \mathrm{mL} \mathrm{X}$-gal (Invitrogen) in a solution of $5 \mathrm{mM}$ potassium ferricyanide (Sigma), $5 \mathrm{mM}$ potassium hexacyanoferrate(II) trihydrate (Sigma), and $2 \mathrm{mM}$ magnesium chloride (Sigma) in PBS for $24 \mathrm{~h}$ at $37^{\circ} \mathrm{C}$.

Corticospinal neuron identification with retrograde neuronal tracers. To identify corticospinal neurons, retrograde neuronal tracers were injected into the spinal cord. Mice were anesthetized with ketamine $(50 \mathrm{mg} / \mathrm{kg})$, dexmedetomidine $(25 \mu \mathrm{m} / \mathrm{kg})$ and the inhalation anesthetic, isoflurane $(0.5-3 \%)$. A small laminectomy was performed over the left cervical cord. After removing one vertebra (C5-C6), 100-200 nl of retrograde neuronal tracer, either green Retrobeads (Lumafluor) or Alexa Fluor 488-conjugated cholera toxin B (Invitrogen), were pressure injected through a glass pipet (20-30 $\mu \mathrm{m}$ tip, inner diameter, Drummond), $500 \mu \mathrm{m}$ lateral to the midline of the exposed spinal cord at a depth of $1000 \mu \mathrm{m}$. Buprenorphine $(0.05 \mathrm{mg} / \mathrm{kg})$ was administered to all animals post-operatively as an analgesic. Mice were sacrificed 7-12 days after tracer injections and brain sections were cut and processed as described above.

Image acquisition and analysis. To assess the overall distribution of C9orf 72 promoter activity, fluorescence images were collected on an AxioImager M1 microscope (Carl Zeiss). Intensity plots for DAPI, $\beta$-gal, and ChAT were made by averaging the signal intensity along the horizontal axis using ImageJ software (NIH). To analyze the distribution of C9orf72 promoter activity within cell types, fluorescence images were acquired with an LSM 510 Meta confocal microscope (Carl Zeiss). Stacks of confocal images ( $0.3 \mu \mathrm{m} z$-interval) were imported into Imaris software for three-dimensional analysis. Surface renderings of NeuN-positive, ChAT-positive, PV-positive, calbindin-positive, Iba1-positive, CC1-positive, NG2-positive, and S100 3 -positive/Olig2-negative cells were created, the locations of $\beta$-gal-positive puncta were marked using the Spots function, and any cell with one or more $\beta$-gal-positive puncta within the three-dimensional rendering was counted as exhibiting C9orf72 expression. As the cerebellar granule cells were very tightly packed, there were occasionally small groups of NeuN-positive 
neurons that could not be clearly segmented into individual neurons. These groups were eliminated from the analysis. To determine whether the distribution of $\beta$-gal puncta related to the underlying cellular distribution, the channel containing the $\beta$-gal signal was flipped horizontally while leaving the cell type-specific channels unchanged. The same counting procedure was then applied to this new configuration.

Analysis of gene expression. The transcript analysis was performed using RNA-sequencing (RNA-seq) data from a previously published study ${ }^{28}$. The RNA-seq data were derived from spinal cords of 3-month old wild-type and C9orf72 (3110043O21Rik) knockout mice (three animals from each group). These knockout mice and the independent line analyzed in the present study were generated from a common source of mouse embryonic stem cells (DEPD00552, the Mouse Biology Program, www.mousebiology.org). The RNA-seq reads were aligned to mouse genome build mm10 using HISAT2 using standard parameters ${ }^{57}$. The BAM files were then visualized using integrated genome viewer ${ }^{58}$. The Sashimi plot was generated to visualize the RNA alignment as well as the splice junctions with a minimum junction coverage of 2 .

Statistics. The Chi-Square test was used to determine whether there was a significant difference in the expected frequencies of $\beta$-gal immunoreactive cells between cell types or between different regions. For the flipped image test, a paired $t$-test was used after confirming the normality of data distribution with the Shapiro Wilk normality test. $p<0.05$ was considered to be statistical significant, and the asterisk was used to mark the statistical significance on the graphs, $*$ for $p<0.05, * *$ for $p<0.01$, and $* * *$ for $p<0.001$.

\section{References}

1. DeJesus-Hernandez, M. et al. Expanded GGGGCC hexanucleotide repeat in noncoding region of C9ORF72 causes chromosome 9p-linked FTD and ALS. Neuron 72, 245-256 (2011).

2. Renton, A. E. et al. A hexanucleotide repeat expansion in C9ORF72 is the cause of chromosome 9p21-linked ALS-FTD. Neuron 72 , 257-268 (2011)

3. Majounie, E. et al. Frequency of the C9orf72 hexanucleotide repeat expansion in patients with amyotrophic lateral sclerosis and frontotemporal dementia: a cross-sectional study. Lancet Neurol 11, 323-330 (2012).

4. Byrne, S. et al. Cognitive and clinical characteristics of patients with amyotrophic lateral sclerosis carrying a C9orf72 repeat expansion: a population-based cohort study. Lancet Neurol 11, 232-240 (2012).

5. Devenney, E. et al. Frontotemporal dementia associated with the C9ORF72 mutation: a unique clinical profile. JAMA Neurol 71, 331-339 (2014)

6. Kohli, M. A. et al. Repeat expansions in the C9ORF72 gene contribute to Alzheimer's disease in Caucasians. Neurobiol Aging 34(1519), e1515-1512 (2013).

7. Majounie, E. et al. Repeat expansion in C9ORF72 in Alzheimer's disease. N Engl J Med 366, 283-284 (2012).

8. Rollinson, S. et al. Analysis of the hexanucleotide repeat in C9ORF72 in Alzheimer's disease. Neurobiol Aging 33(1846), e1845-1846 (2012).

9. Harms, M. et al. C9orf72 hexanucleotide repeat expansions in clinical Alzheimer disease. JAMA Neurol 70, 736-741 (2013).

10. Lesage, S. et al. C9orf72 repeat expansions are a rare genetic cause of parkinsonism. Brain 136, 385-391 (2013).

11. Hensman Moss, D. J. et al. C9orf72 expansions are the most common genetic cause of Huntington disease phenocopies. Neurology 82, 292-299 (2014).

12. Kostic, V. S., Dobricic, V., Stankovic, I., Ralic, V. \& Stefanova, E. C9orf72 expansion as a possible genetic cause of Huntington disease phenocopy syndrome. J Neurol 261, 1917-1921 (2014).

13. Galimberti, D. et al. C9ORF72 hexanucleotide repeat expansion as a rare cause of bipolar disorder. Bipolar Disord 16, 448-449 (2014).

14. Goldman, J. S. et al. Multiple system atrophy and amyotrophic lateral sclerosis in a family with hexanucleotide repeat expansions in C9orf72. JAMA Neurol 71, 771-774 (2014).

15. Bieniek, K. F. et al. Expanded C9ORF72 hexanucleotide repeat in depressive pseudodementia. JAMA Neurol 71, 775-781 (2014).

16. Galimberti, D. et al. C9ORF72 hexanucleotide repeat expansion is a rare cause of schizophrenia. Neurobiol Aging 35, $1214 \mathrm{e} 1217-$ $1214 \mathrm{e} 1210,(2014)$.

17. Taylor, J. P., Brown, R. H. Jr. \& Cleveland, D. W. Decoding ALS: from genes to mechanism. Nature 539, 197-206 (2016).

18. Haeusler, A. R., Donnelly, C. J. \& Rothstein, J. D. The expanding biology of the C9orf72 nucleotide repeat expansion in neurodegenerative disease. Nat Rev Neurosci 17, 383-395 (2016).

19. Gijselinck, I. et al. A C9orf72 promoter repeat expansion in a Flanders-Belgian cohort with disorders of the frontotemporal lobar degeneration-amyotrophic lateral sclerosis spectrum: a gene identification study. Lancet Neurol 11, 54-65 (2012).

20. Belzil, V. V. et al. Reduced C9orf72 gene expression in c9FTD/ALS is caused by histone trimethylation, an epigenetic event detectable in blood. Acta Neuropathol 126, 895-905 (2013).

21. Waite, A. J. et al. Reduced C9orf72 protein levels in frontal cortex of amyotrophic lateral sclerosis and frontotemporal degeneration brain with the C9ORF72 hexanucleotide repeat expansion. Neurobiol Aging 35, 1779 e1775-1779 e1713, (2014).

22. Liu, E. Y. et al. C9orf72 hypermethylation protects against repeat expansion-associated pathology in ALS/FTD. Acta Neuropathol 128, 525-541 (2014).

23. Rizzu, P. et al. C9orf72 is differentially expressed in the central nervous system and myeloid cells and consistently reduced in C9orf72, MAPT and GRN mutation carriers. Acta Neuropathol Commun 4, 37 (2016).

24. Atanasio, A. et al. C9orf72 ablation causes immune dysregulation characterized by leukocyte expansion, autoantibody production, and glomerulonephropathy in mice. Sci Rep 6, 23204 (2016).

25. Burberry, A. et al. Loss-of-function mutations in the C9ORF72 mouse ortholog cause fatal autoimmune disease. Sci Transl Med 8 , $347 \mathrm{ra3} 3$ (2016).

26. Jiang, J. et al. Gain of toxicity from ALS/FTD-linked repeat expansions in C9orf72 Is alleviated by antisense oligonucleotides targeting GGGGCC-containing RNAs. Neuron 90, 535-550 (2016).

27. Koppers, M. et al. C9orf72 ablation in mice does not cause motor neuron degeneration or motor deficits. Ann Neurol 78, 426-438 (2015).

28. O'Rourke, J. G. et al. C9orf72 is required for proper macrophage and microglial function in mice. Science 351, 1324-1329 (2016).

29. Ugolino, J. et al. Loss of C9orf72 enhances autophagic activity via deregulated mTOR and TFEB signaling. PLoS Genet 12, e1006443 (2016).

30. Peters, O. M. et al. Human C9ORF72 Hexanucleotide Expansion Reproduces RNA Foci and Dipeptide Repeat Proteins but Not Neurodegeneration in BAC Transgenic Mice. Neuron 88, 902-909 (2015).

31. Liu, Y. et al. C9orf72 BAC mouse model with motor deficits and neurodegenerative features of ALS/FTD. Neuron 90, 521-534 (2016). 
32. Suzuki, N. et al. The mouse C9ORF72 ortholog is enriched in neurons known to degenerate in ALS and FTD. Nat Neurosci 16, 1725-1727 (2013).

33. Pham, C. T., MacIvor, D. M., Hug, B. A., Heusel, J. W. \& Ley, T. J. Long-range disruption of gene expression by a selectable marker cassette. Proc Natl Acad Sci USA 93, 13090-13095 (1996).

34. Rijli, F. M., Dolle, P., Fraulob, V., LeMeur, M. \& Chambon, P. Insertion of a targeting construct in a Hoxd-10 allele can influence the control of Hoxd-9 expression. Dev Dyn 201, 366-377 (1994).

35. Hayashi, S., Lewis, P., Pevny, L. \& McMahon, A. P. Efficient gene modulation in mouse epiblast using a Sox2Cre transgenic mouse strain. Gene Expr Patterns 2, 93-97 (2002).

36. Prieto, J. J., Peterson, B. A. \& Winer, J. A. Morphology and spatial distribution of GABAergic neurons in cat primary auditory cortex (AI). J Comp Neurol 344, 349-382 (1994).

37. Tremblay, R., Lee, S. \& Rudy, B. GABAergic interneurons in the neocortex: from cellular properties to circuits. Neuron 91, 260-292 (2016).

38. Al-Sarraj, S. et al. p62 positive, TDP-43 negative, neuronal cytoplasmic and intranuclear inclusions in the cerebellum and hippocampus define the pathology of C9orf72-linked FTLD and MND/ALS. Acta Neuropathol 122, 691-702 (2011).

39. Mori, K. et al. The C9orf72 GGGGCC repeat is translated into aggregating dipeptide-repeat proteins in FTLD/ALS. Science 339, 1335-1338, (2013).

40. Ash, P. E. et al. Unconventional translation of C9ORF72 GGGGCC expansion generates insoluble polypeptides specific to c9FTD/ ALS. Neuron 77, 639-646 (2013).

41. Gendron, T. F. et al. Antisense transcripts of the expanded C9ORF72 hexanucleotide repeat form nuclear RNA foci and undergo repeat-associated non-ATG translation in c9FTD/ALS. Acta Neuropathol 126, 829-844 (2013).

42. Ferguson, R., Serafeimidou-Pouliou, E. \& Subramanian, V. Dynamic expression of the mouse orthologue of the human amyotropic lateral sclerosis associated gene C9orf72 during central nervous system development and neuronal differentiation. J Anat 229, 871-891 (2016).

43. Valori, C. F., Brambilla, L., Martorana, F. \& Rossi, D. The multifaceted role of glial cells in amyotrophic lateral sclerosis. Cell Mol Life Sci 71, 287-297 (2014).

44. Philips, T. \& Rothstein, J. D. Glial cells in amyotrophic lateral sclerosis. Exp Neurol 262(Pt B), 111-120 (2014).

45. Meyer, K. et al. Direct conversion of patient fibroblasts demonstrates non-cell autonomous toxicity of astrocytes to motor neurons in familial and sporadic ALS. Proc Natl Acad Sci USA 111, 829-832 (2014).

46. Zhang, Y. et al. An RNA-sequencing transcriptome and splicing database of glia, neurons, and vascular cells of the cerebral cortex. J Neurosci 34, 11929-11947 (2014).

47. Zhang, Y. et al. Purification and characterization of progenitor and mature human astrocytes reveals transcriptional and functional differences with mouse. Neuron 89, 37-53 (2016).

48. Funfschilling, U. et al. Glycolytic oligodendrocytes maintain myelin and long-term axonal integrity. Nature 485, 517-521 (2012).

49. Lee, Y. et al. Oligodendroglia metabolically support axons and contribute to neurodegeneration. Nature 487, 443-448 (2012).

50. Philips, T. et al. Oligodendrocyte dysfunction in the pathogenesis of amyotrophic lateral sclerosis. Brain 136, 471-482 (2013).

51. Kang, S. H., Fukaya, M., Yang, J. K., Rothstein, J. D. \& Bergles, D. E. NG2 + CNS glial progenitors remain committed to the oligodendrocyte lineage in postnatal life and following neurodegeneration. Neuron 68, 668-681 (2010).

52. Kang, S. H. et al. Degeneration and impaired regeneration of gray matter oligodendrocytes in amyotrophic lateral sclerosis. Nat Neurosci 16, 571-579 (2013).

53. Henry, A. M. \& Hohmann, J. G. High-resolution gene expression atlases for adult and developing mouse brain and spinal cord. Mamm Genome 23, 539-549 (2012).

54. Geevasinga, N. et al. Cortical function in asymptomatic carriers and patients with C9orf72 amyotrophic lateral sclerosis. JAMA Neurol 72, 1268-1274 (2015).

55. Schanz, O. et al. Cortical hyperexcitability in patients with C9ORF72 mutations: Relationship to phenotype. Muscle Nerve 54, 264-269 (2016)

56. Tan, R. H. et al. Cerebellar neuronal loss in amyotrophic lateral sclerosis cases with ATXN2 intermediate repeat expansions. Ann Neurol 79, 295-305 (2016).

57. Kim, D., Langmead, B. \& Salzberg, S. L. HISAT: a fast spliced aligner with low memory requirements. Nat Methods 12, 357-360 (2015).

58. Robinson, J. T. et al. Integrative genomics viewer. Nat Biotechnol 29, 24-26 (2011).

\section{Acknowledgements}

We thank Dr. Joshua R. Sanes (Harvard University) for providing the anti- $\beta$-gal antibody, and Karen Conchina and Justin Chu for their assistance in the generation and maintenance of the mice. This work was supported by Target ALS (D.E.B., S.P.B.), the Robert Packard Center for ALS Research (J.W., S.P.B.), the U.S. Department of Defense (J.W.) and the National Institutes of Health (J.W.: NS074324 and NS089616; S.P.B.: NS098819; D.E.B.: NS050274). J.K. was supported by a National Research Foundation of Korea Fellowship (NRF-2011-357-E00005). A.J.L. was supported by a National Multiple Sclerosis Society Postdoctoral Fellowship (FG 20114-A-1). S.P.B. is supported by a Klingenstein-Simons Fellowship in the Neurosciences.

\section{Author Contributions}

J.W., D.E.B., and S.P.B. conceived of the project. A.J.L., J.K., D.E.B., and S.P.B. designed the study. J.E.U., Y.S. and J.W. generated the mice. A.J.L. and J.K. acquired and analysed the anatomical data. H.-Y. H. analyzed the RNAsequencing data. A.J.L., J.K., D.E.B., and S.P.B. interpreted the data and drafted the manuscript. J.E.U. and J.W. contributed to the writing of the manuscript.

\section{Additional Information}

Supplementary information accompanies this paper at doi:10.1038/s41598-017-05864-2

Competing Interests: The authors declare that they have no competing interests.

Publisher's note: Springer Nature remains neutral with regard to jurisdictional claims in published maps and institutional affiliations. 
(i) Open Access This article is licensed under a Creative Commons Attribution 4.0 International License, which permits use, sharing, adaptation, distribution and reproduction in any medium or format, as long as you give appropriate credit to the original author(s) and the source, provide a link to the Creative Commons license, and indicate if changes were made. The images or other third party material in this article are included in the article's Creative Commons license, unless indicated otherwise in a credit line to the material. If material is not included in the article's Creative Commons license and your intended use is not permitted by statutory regulation or exceeds the permitted use, you will need to obtain permission directly from the copyright holder. To view a copy of this license, visit http://creativecommons.org/licenses/by/4.0/.

(C) The Author(s) 2017 